Trauma Berufskrankh 2010 · 12[Suppl 3]:273-278 DOI 10.1007/s10039-010-1602-2

Online publiziert: 3. April 2010

(c) Springer-Verlag 2010
J. Beller · D. Maier $\cdot$ M. Arand $\cdot$ T. Geyer

Klinik für Unfall-, Wiederherstellungschirurgie und Orthopädie, Klinikum Ludwigsburg, Akademisches Lehrkrankenhaus, Universität Heidelberg, Ludwigsburg

\title{
Behandlungsstrategie bei distalen Humerusfrakturen
}

\section{Epidemiologie}

Die distale Humerusfraktur des Erwachsenen ist mit einer Inzidenz von 5/100.00o Einwohnern und 2-6\% aller Frakturen eine relativ seltene Bruchverletzung. In der Gesamtheit aller Ellenbogenfrakturen nimmt sie einen Anteil von $30 \%$ ein. In der alters- und geschlechtsspezifischen Verteilung zeigen sich Häufungen bei jüngeren Männern und älteren Frauen. Bei den Frakturen älterer Menschen tritt eine Häufung von komplizierten artikulären Brüchen unter Ein- beziehung des ulnaren und radialen Kondylus bei simultaner Reduktion der Montagestabilität auf.

Entsprechend der AO-Klassifikation (AO: Arbeitsgemeinschaft Osteosynthesefragen; 0 Tab. 1, Abb. 3) sind

- $38 \%$ als Typ-A-Fraktur,

- $24 \%$ als Typ-B-Fraktur und

- $37 \%$ als Typ-C-Fraktur

anzusehen [12].

Der hohe Schweregrad der distalen Humerusfraktur wird deutlich bei einem Anteil von bis zu $40 \%$ offenen Frakturen
[10] sowie einer Häufigkeit von begleitenden primären Nervenverletzungen von $20 \%$ [7] und arteriellen Gefäßverletzungen von $5-10 \%$. [9]

Als Verletzungsursache im gesamten Patientenkollektiv steht der einfache Sturz im Vordergrund, gefolgt von Verkehrsund Sportunfällen. In einer In-vitro-Studie mit Ellenbogengelenken von Leichen konnten Amis u. Miller [1] zeigen, dass distale Humerusfrakturen v. a. beim Sturz auf den über $110^{\circ}$ gebeugten Ellenbogen entstehen.

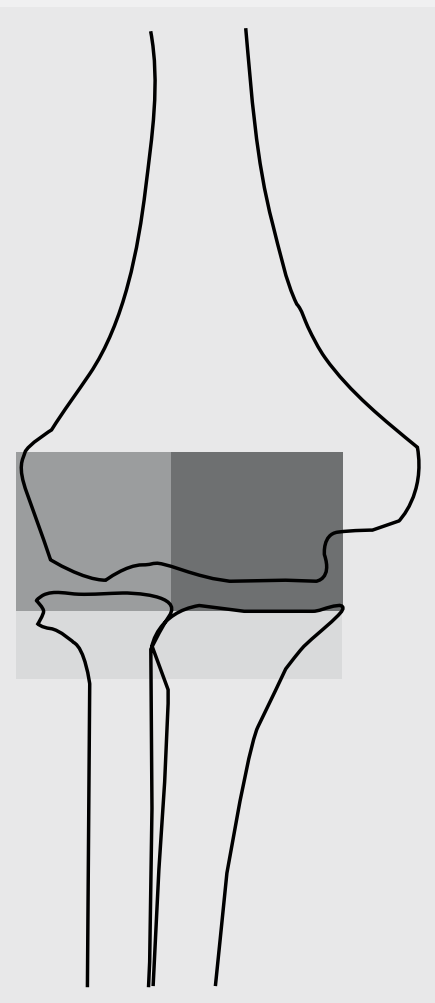

Abb. 14 Ellenbogengelenk, dunkelgrau Articulatio humeroulnaris, mittelgrau Articulatio humeroradialis, hellgrau proximales Radioulnargelenk

Abb. 2 Abduktionswinkel zwischen Oberund Unterarmachse, palmar vorverlagertes Rotationszentrum
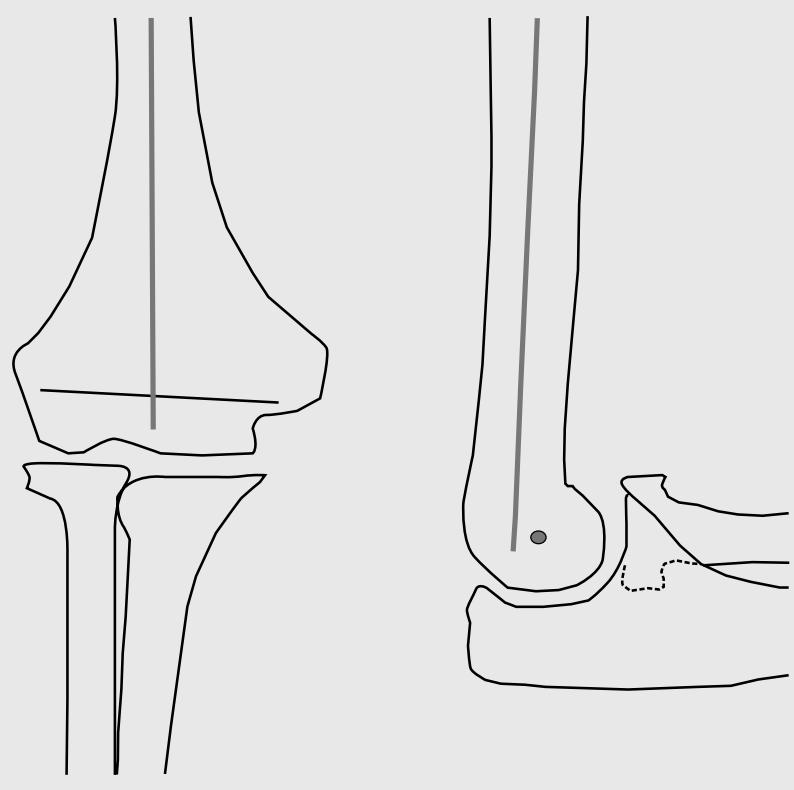


\section{Ellenbogengelenk}

1
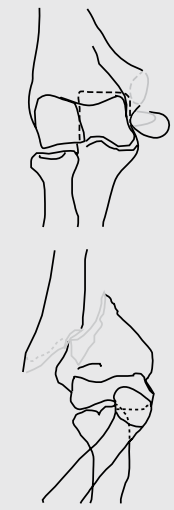

1

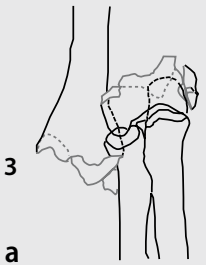

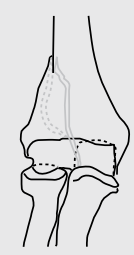

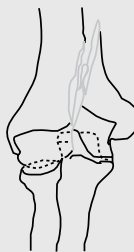

b

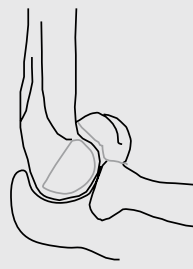

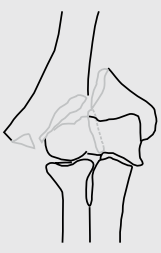
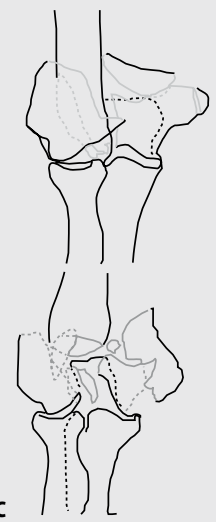

Abb. $3 \Delta$ AO-Klassifikation der distalen Humerusfraktur, a Typ A, b Typ B, c Typ C. (Mit freundl. Genehmigung der AO Foundation, Davos, Schweiz)

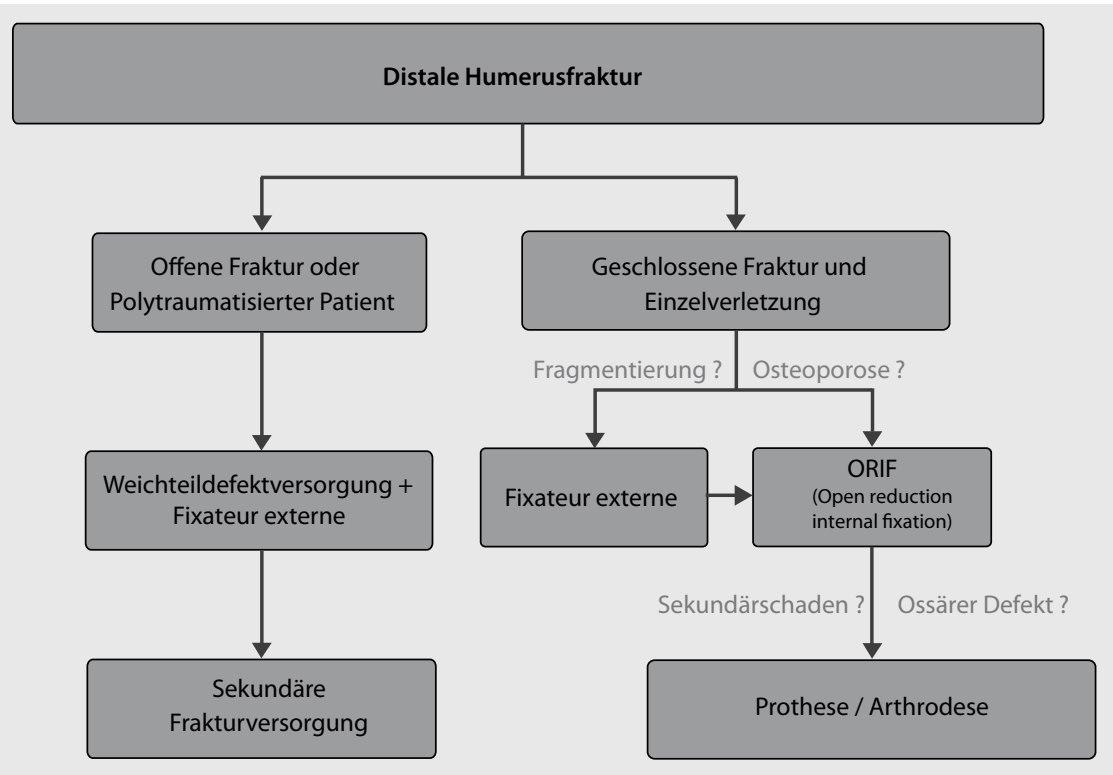

Abb. 4 \ Algorithmus zur operativen Versorgung einer distalen Humerusfraktur

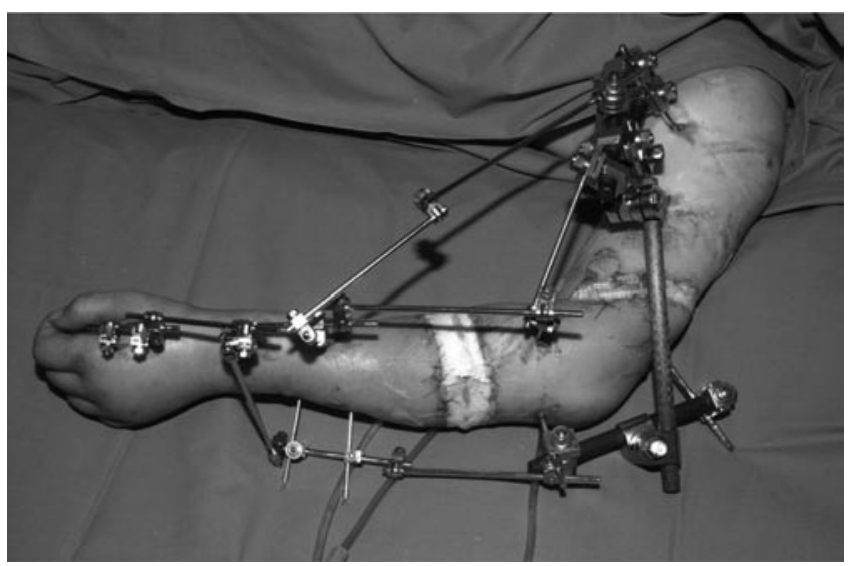

Abb. $5<$ Primäre Versorgung einer distalen Humerusfraktur mit schweren Kombinationsverletzungen am Weichteilmantel und knöchernen Unterarm

\section{Anatomie}

Das Ellenbogengelenk setzt sich aus der Articulatio humeroulnaris, der Articulatio humeroradialis und dem proximalen Radioulnargelenk zusammen (• Abb. 1).

Es bestehen enge topographische Beziehungen der knöchernen Strukturen zu den angrenzend verlaufenden Leitungsbahnen A. und V. brachialis sowie N. medianus, $\mathrm{N}$. ulnaris und $\mathrm{N}$. radialis.

Der mediale und der laterale Pfeiler des distalen Humerus bilden mit der dazwischen liegenden Trochlea ein stabiles Dreieck. Die Gelenk bildende Komponente der medialen Säule stellt die Trochlea humeri, die der lateralen Säule das Capitulum humeri dar. Die dünne Knochenlamelle zwischen der medialen und der lateralen Säule bildet den jeweiligen Boden der beugeseitigen Fossa coronoidea sowie der dorsalen Fossa olecrani.

Die knöcherne Achse zwischen Oberund Unterarm weist im Mittel einen Abduktionswinkel von $168,5^{\circ}$ auf. Die Rotationsachse des Ellenbogengelenks ist im Vergleich zur Humeruslängsachse durch die beugeseitige Kippung von Trochlea und Capitulum nach palmar verlagert

\section{(- Abb. 2).}

Die Lastverteilung beim gestreckten Ellenbogengelenk zwischen radial und ulnar weist ein Verhältnis von 3:2 auf [14], was die Notwendigkeit der stabilen Rekonstruktion beider Säulen verdeutlicht.

\section{Diagnostik}

Im Rahmen der klinischen Untersuchung ist insbesondere auf den Pulsstatus, mögliche neurologische Defizite sowie den Zustand des umgebenden Weichteilmantels zu achten. Bei nicht palpablen Pulsen sollten eine dopplersonographische Untersuchung und ggf. eine Angiographie durchgeführt werden.

In der bildgebenden Diagnostik ist zunächst ein konventionelles Röntgenbild in 2 Ebenen anzufertigen. Zum besseren Verständnis der Frakturmorphologie und für die präoperative Planung ist häufig die Durchführung einer Computertomographie sinnvoll. 


\section{Klassifikation}

In der geläufigen $\mathrm{AO}-$ Klassifikation der distalen Humerusfraktur nach Müller werden 3 Frakturtypen unterschieden (- Tab. 1, A Abb. 3).

\section{Therapie}

\section{Konservative Behandlung}

Sie sollte nur bei Vorliegen von schwerwiegenden Kontraindikationen oder undislozierten Frakturen älterer Patienten als Option erwogen werden. Bedingt durch die notwendige 6- bis 8-wöchige Ruhigstellung sind massive Bewegungseinschränkungen in aller Regel unvermeidbar.

\section{Operative Therapieoptionen}

Die offene Reposition und Osteosynthese der distalen Humerusfraktur des Erwachsenen gilt in der heutigen Zeit als Goldstandard [11]. Der klinische Behandlungsalgorithmus zur operativen Versorgung dieser Verletzung ist in $\mathbf{A b b .} 4$ grafisch dargestellt.

Bei Vorliegen einer offenen Fraktur oder beim polytraumatisierten $\mathrm{Pa}$ tienten erfolgen primär nur die Versorgung des Weichteildefektes mittels primärem Wundverschluss oder Vakuumversiegelung und die Anlage eines Fixateur externe (■ Abb. 5). Hierfür werden jeweils 2 Schanz-Schrauben in den lateralen oder alternativ dorsalen Humerus und die dorsoradiale Ulna eingebracht und diese dann mit einem Gelenk überbrückenden Stabsystem verbunden. Hierbei ist auf die Schonung des $\mathrm{N}$. radialis zu achten, bei lateraler Platzierung sind die Pins ausreichend proximal zu setzen.

Ziel der operativen Versorgung müssen neben der Wiederherstellung des Weichteilmantels die stabile Verbindung zwischen Epiphyse und Diaphyse sowie eine möglichst passgenaue Rekonstruktion der Gelenkfläche sein. Durch die damit ermöglichte funktionelle Nachbehandlung wird die größtmögliche Funktionalität des Ellenbogengelenks bewahrt. Eine exakte Rekonstruktion der gelenkbildenden Fragmente ist auch im Sinne der Prophylaxe einer posttraumatischen Arthrose anzustreben.
Tab. 1 A0-Klassifikation der distalen Humerusfraktur

\begin{tabular}{|lll}
\hline Typ & Charakteristika & \\
A & Extraartikuläre Frakturen & Abb. 3a \\
\hline B & $\begin{array}{l}\text { Partielle (monokondyläre) } \\
\text { Gelenkfrakturen }\end{array}$ & Abb. 3b \\
\hline C & $\begin{array}{l}\text { Vollständige (bikondyläre) } \\
\text { Gelenkfrakturen }\end{array}$ & Abb. 3c \\
\hline
\end{tabular}

\section{Zugang}

Typ-A-und-B-Frakturen. Extraartikuläre (Typ A) oder monokondyläre Frakturen (Typ B) können entsprechend ihrer Lokalisation über einen radialen oder ulnaren Zugang versorgt werden. Diese Operation kann in Rückenlage durchgeführt werden. Beim ulnaren Zugang ist die intraoperative Darstellung des N. ulnaris obligat. Während isolierte Abrissfrakturen des Epikondylus (Typ A1) und einzelne monokondyläre Frakturen (Typ B3) über eine Schraubenosteosynthese inklusive Rotationssicherung adäquat versorgt werden können, ist bei allen anderen Frakturtypen eine Plattenosteosynthese zu empfehlen (- Abb. 6).

Typ-C-Frakturen. Insbesondere bei bikondylären Gelenkfrakturen (Typ C) ist eine gute Einsicht in das Gelenk erforderlich. Daher sollten diese über einen dorsalen Zugang mit Olekranonosteotomie angegangen werden [2]. Der Patient wird in Bauchlage mit hängendem Arm gelagert. Das Ellenbogengelenk muss bis $120^{\circ}$ flektierbar sein. Man wählt den Standardzugang nach Kocher dorsalseitig unter radialseitiger Umschneidung des Olekranons.

Im ersten Schritt muss der N. ulnaris in seinem Verlauf dargestellt und angeschlungen werden (• Abb. 7, Foto 1). Nach keilförmiger (Chevron-)Osteotomie des Olekranons mittels oszillierender Säge und Durchtrennung der Gegenkortikalis mit dem Klingenmeißel (- Abb. 7, Foto 2) wird der M. triceps unter Schonung des N. ulnaris von der Rückseite des Humerus präpariert. Nach Hochklappen des Olekranons im Verbund mit dem M. triceps brachii wird das Ausmaß der Gelenkdestruktion sichtbar ( Abb. 7, Foto 3).

In der Regel wird zunächst der Gelenkblock rekonstruiert, in Einzelfällen kann jedoch in Abhängigkeit vom Zerstörungsgrad der primäre Aufbau eines rekonstru-
Trauma Berufskrankh 2010 - 12[Suppl 3]:

273-278

DOI 10.1007/s10039-010-1602-2

(C) Springer-Verlag 2010

J. Beller $\cdot$ D. Maier $\cdot$ M. Arand $\cdot$ T. Geyer

\section{Behandlungsstrategie bei distalen Humerusfrakturen}

\section{Zusammenfassung}

Die operative Versorgung distaler Humerusfrakturen stellt aufgrund der komplexen Gelenkstruktur und der anatomischen Nähe zwischen Knochen, Nerven und Gefäßen eine Herausforderung in der Unfallchirurgie dar. Ihr Ziel sollten neben der Wiederherstellung des Weichteilmantels die stabile Verbindung zwischen Epiphyse und Diaphyse sowie eine möglichst passgenaue Rekonstruktion der Gelenkfläche sein. Das Erreichen einer übungsstabilen Osteosynthese ist die Voraussetzung, um über eine frühfunktionelle Nachbehandlung ein gutes Endergebnis erzielen zu können.

\section{Schlüsselwörter}

Distale Humerusfraktur · Operative Versorgung · Passgenaue Rekonstruktion . Übungsstabile Osteosynthese. Frühfunktionelle Nachbehandlung

\section{Treatment strategy for distal humerus fractures}

\section{Abstract}

Surgical treatment of distal humerus fractures represents a challenge in traumatology due to the complex joint structure and the anatomical proximity of bone, nerves and blood vessels. The aim of surgical treatment should be the anatomical reconstruction of the articular surface, the creation of a stable interface between diaphysis and epiphysis and reconstitution of the surrounding soft tissue. Achieving exercise-stable internal fixation is an absolute requirement for early functional rehabilitation to accomplish a satisfactory result.

\section{Keywords}

Distal humerus fracture - Surgical treatment . Anatomical reconstruction. Excercise-stable internal fixation . Early functional rehabilitation 


\section{Ellenbogengelenk}
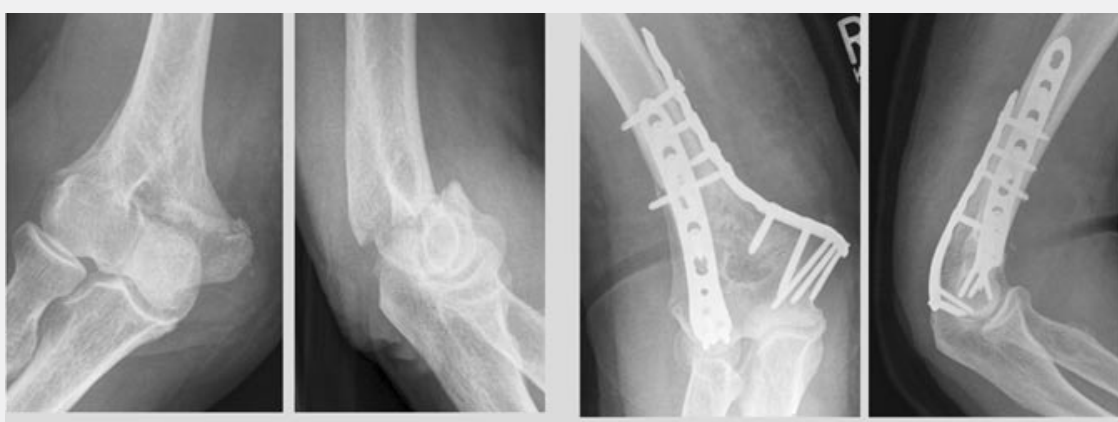

\section{A2-Fraktur}
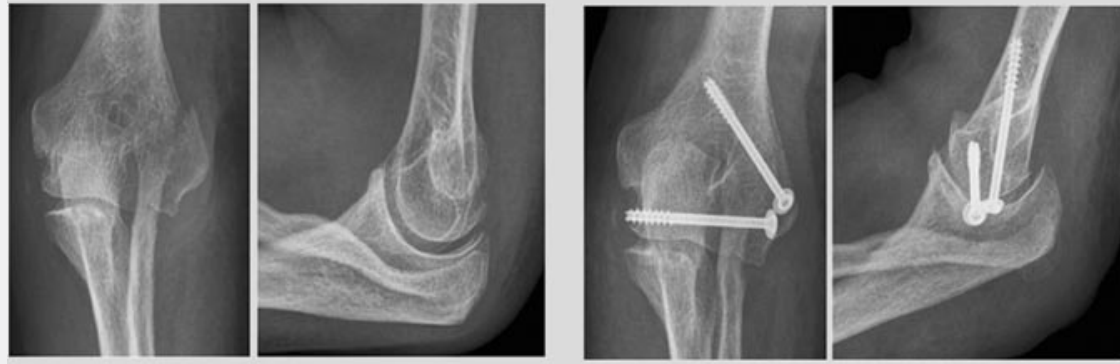

B1-Fraktur
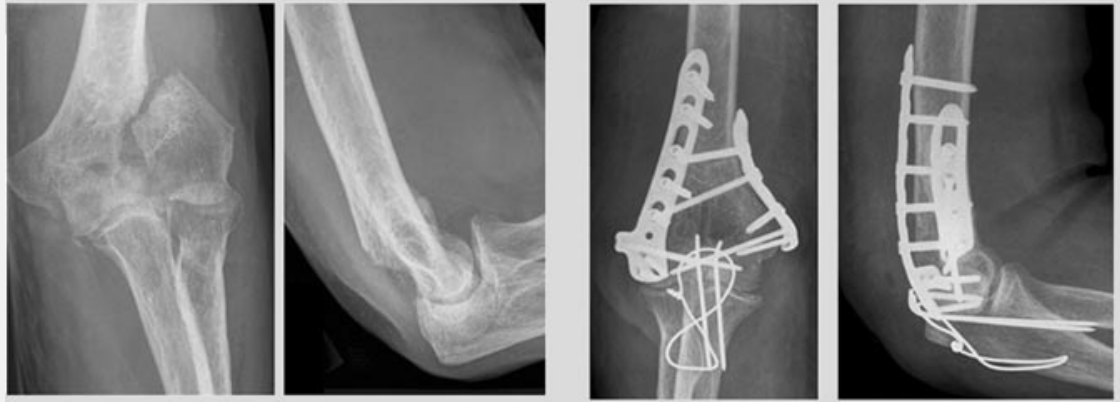

C1-Fraktur

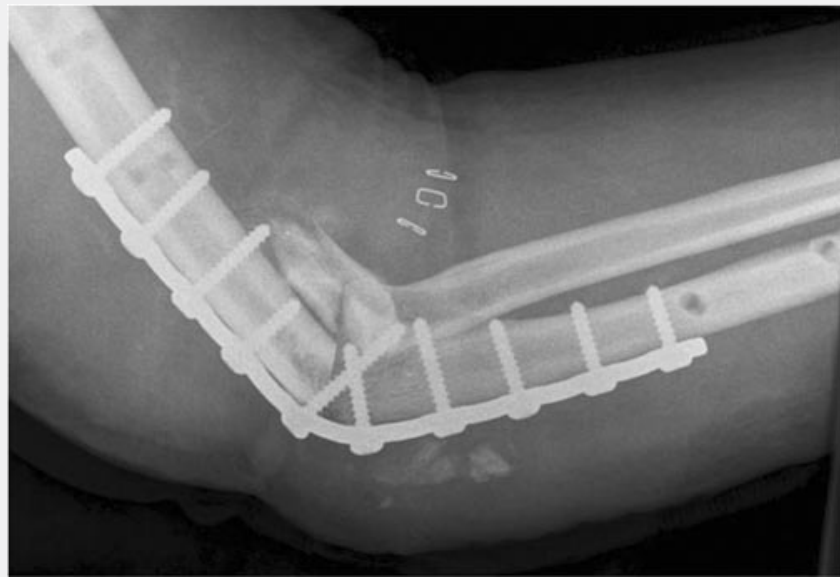

Abb. $8 \Delta$ Arthrodese bei drittgradig offener subtotaler Amputationsverletzung und schwerem Schädel-Hirn-Trauma

Abb. 9 Ellenbogenprothesenimplantation nach fehlgeschlagener vorangegangener Osteosynthese

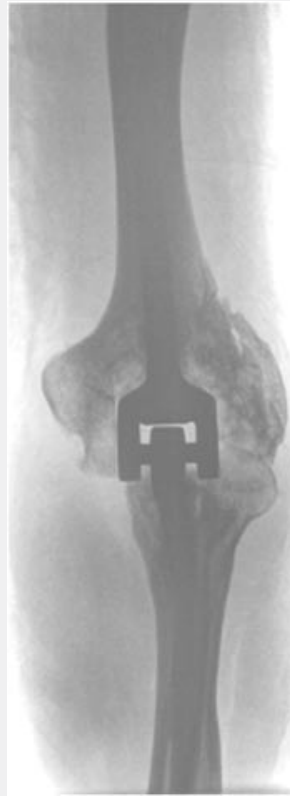

Abb. 64 Beispiele von distalen Humerusfrakturen mit postoperativem Ergebnis: A2-Fraktur mit winkelstabiler Formplattenosteosynthese ohne Notwendigkeit einer Olekranonosteotomie, B1-Fraktur mit Spongiosaschraubenosteosynthese, C1-Fraktur mit winkelstabiler Form-plattenosteosynthese und Olekranonosteotomie 


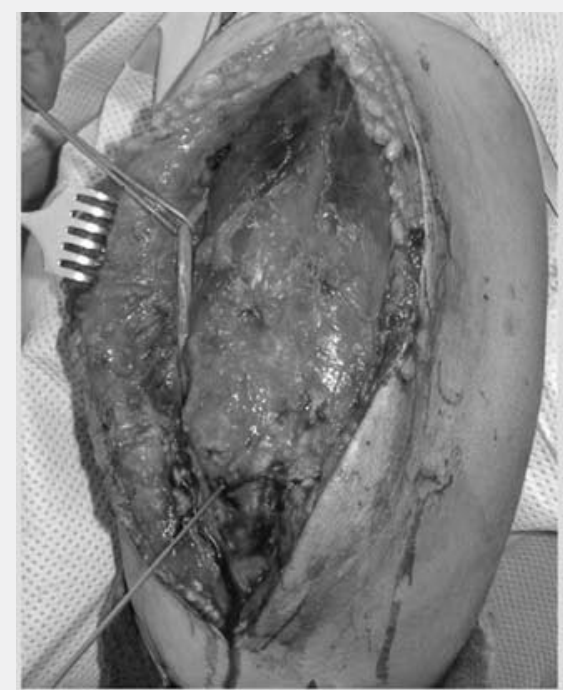

Foto 1

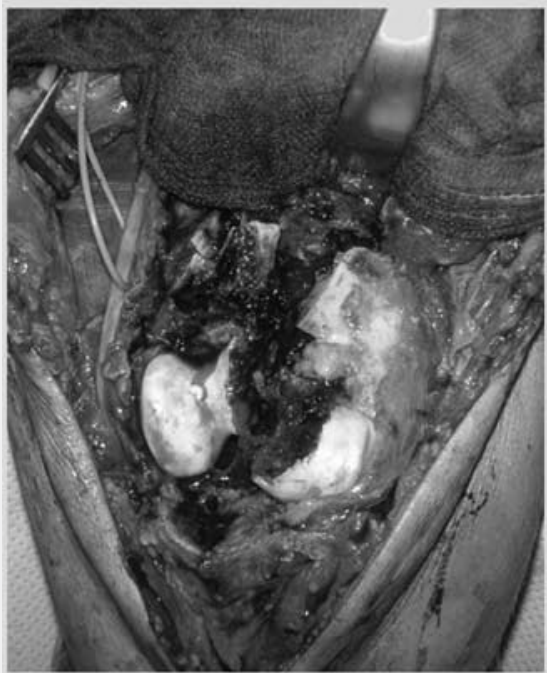

Foto 3

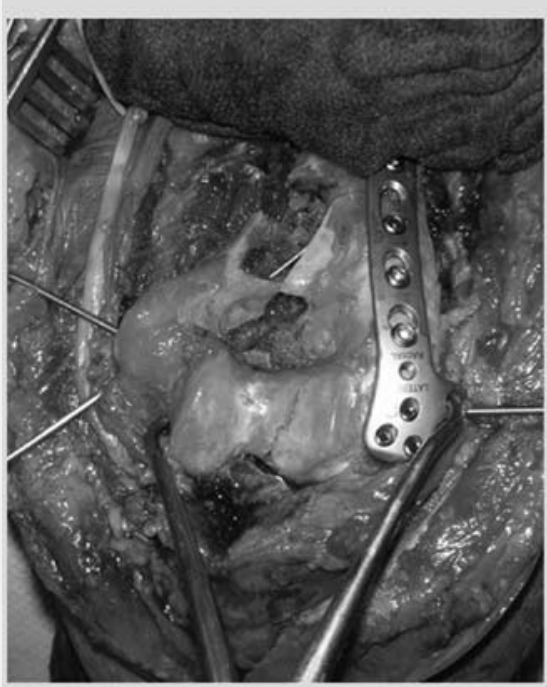

Foto 5

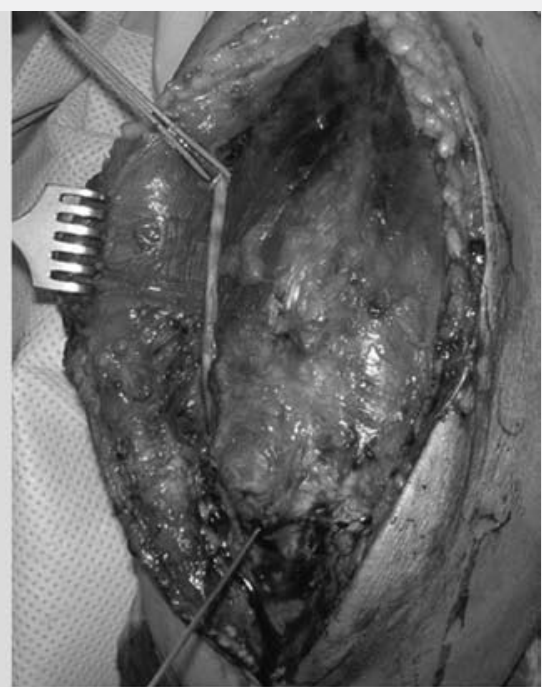

Foto 2

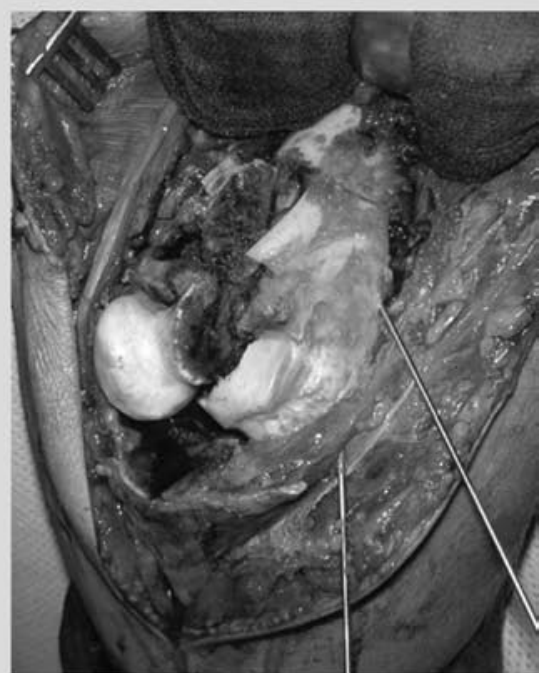

Foto 4

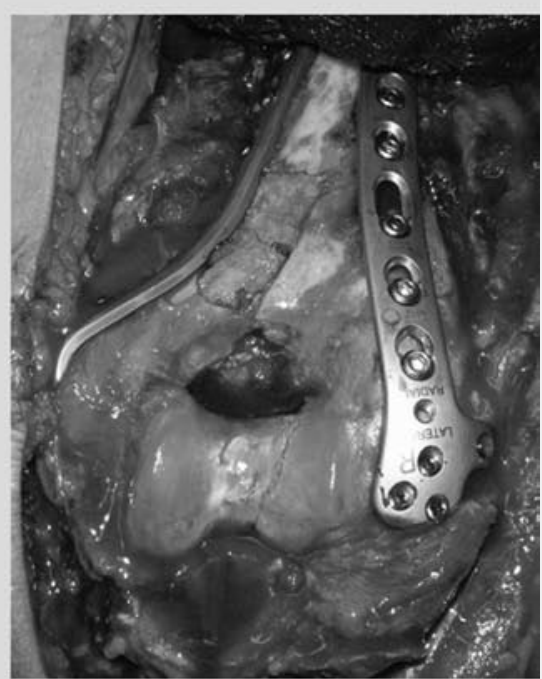

Foto 6 
ierbaren Pfeilers sinnvoll sein (• Abb. 7, Foto 4). Die Rekonstruktion des Gelenkblocks und die Fixation erfolgen mittels Schrauben oder präliminarer Kirschner-Drähte. Bei Knochenverlust wird eine Spaninterposition aus dem Beckenkamm vorgenommen. Für die Retention sind beide Humeruspfeiler mit einer stabilen Plattenosteosynthese zu versorgen. Hierfür besonders gut geeignete Implantate sind winkelstabile anatomisch vorgeformte Platten ( $\bullet$ Abb. 7, Foto 5). Auf dem ulnaren Pfeiler sollte eine medialseitige, auf dem radialen Pfeiler eine dorsalseitige Plattenlage angestrebt werden ([4],

- Abb. 7, Foto 6).

Abschließend erfolgt die Osteosynthese der Olekranonosteotomie mittels Zuggurtung oder langer GF-Spongiosaschraube (GF: Großfragment).

\section{Nachbehandlung}

Das Erreichen einer übungsstabilen Osteosynthese ist für die erfolgreiche Nachbehandlung essenziell. Bei Vorliegen schwieriger Weichteilverhältnisse kann bis zum Abschluss der Wundheilung eine Oberarmschiene angelegt werden. Bereits wenige Tage postoperativ sollte mit der passiven physiotherapeutischen Übungsbehandlung begonnen werden. Nach 3 Wochen können zunehmend auch aktive Übungen durchgeführt werden. Die volle Belastbarkeit wird nach etwa 12 Wochen erreicht.

Weitere Röntgenverlaufskontrollen werden nach 3, 6 und 12 Wochen durchgeführt.

\section{Operative Rescue-Verfahren}

Bei ausgeprägtem primärem Defekt oder bei eingetretenem Sekundärschaden stehen als operative Versorgungsmöglichkeiten die Implantation einer Ellenbogengelenkprothese [3] oder die Arthrodese des Gelenks zu Verfügung (• Abb. 8).

Weber et al. [13] zeigten in einer aktuellen Studie über die primäre Versorgung des frakturierten Ellenbogens beim hochbetagten Patienten gute funktionelle Ergebnisse bei der primären Durchführung einer Alloarthoplastik mittels Implantation einer zementierten CoonradMorrey-Prothese. Dennoch sehen wir, insbesondere aufgrund der Möglichkeit der Anwendung winkelstabiler Formplat- ten, die Indikation zum Einsatz der Ellenbogenendoprothese überwiegend in der Sekundärversorgung bei Vorliegen einer posttraumatischen Arthrose oder bei fehlgeschlagener primärer Osteosynthese (- Abb. 9).

\section{Komplikationen und Prognose}

Postoperative Nervenschäden betreffen überwiegend den N. ulnaris mit einer Inzidenz von $7-15 \%$ der Fälle [5]. In der Regel zeigt sich hierbei ein spontan reversibler Verlauf.

Das Auftreten von periartikulären Ossifikationen ist in der Literatur in bis zu 49\% der Fälle beschrieben [8], wobei zwischen lediglich radiologisch sichtbaren und funktionell relevanten Ossifikationen unterschieden werden muss. Prophylaktisch sollte daher ein NSAR (nichtsteroidales Antirheumatikum, z. B. Indometacin) über einen Zeitraum von 2 Wochen verabreicht werden.

Die Pseudarthroserate liegt unter 10\%, die postoperative Infektrate wird je nach Literaturzitat mit etwa 5-10\% [6] angegeben. Das Auftreten einer posttraumatische Arthrose ist in der Literatur in bis zu 80\% der Fälle beschrieben [6].

\section{Fazit}

Die operative Versorgung der distalen Humerusfraktur erfolgt bei geschlossener Fraktur und nicht polytraumatisiertem Patienten durch die frühzeitige offene Reposition und Osteosynthese. Als besonders hilfreich bei komplexen Frakturen haben sich winkelstabile Platten und anatomisch vorgeformte Implantate erwiesen, die auch bei osteoporotischem Knochen eine übungsstabile Osteosynthese erlauben.

Bei offenen Frakturen oder beim polytraumatisierten Patienten sollten zunächst eine Ruhigstellung der Fraktur im Fixateur externe sowie eine adäquate Weichteilversorgung angestrebt werden, um dann bei verbesserter Gesamtsituation eine sekundäre Osteosynthese durchführen zu können.

Nur eine übungsstabile Osteosynthese erlaubt die frühfunktionelle Bewegungstherapie, die zum Erreichen eines guten Ergebnisses unerlässlich ist.
Bei ausgeprägter Destruktion und fehlender Rekonstruktionsmöglichkeit scheint auch die primäre Implantation einer Ellenbogengelenkprothese gute Ergebnisse erzielen zu können. Ansonsten sind die Alloarthoplastik sowie die Arthrodese des Ellenbogens als eine Therapieoption bei eingetretenem Sekundärschaden zu betrachten.

\section{Korrespondenzadresse}

Dr. J. Beller

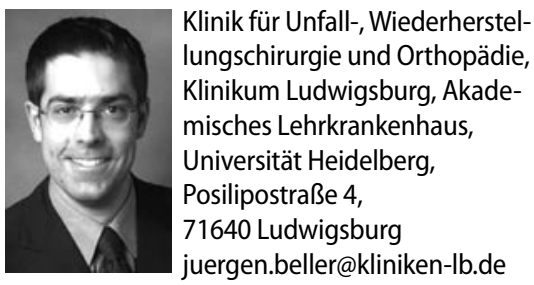

Interessenkonflikt. Der korrespondierende Autor gibt an, dass kein Interessenkonflikt besteht.

\section{Literatur}

1. Amis AA, Miller JH (1995) The mechanisms of elbow fractures: an investigation using impact tests in vitro. Injury 26(3):163-168

2. De Boer P, Stanley D (1998) Surgery of the elbow: practical and scientific aspects. Surgical approaches to the elbow. Arnold, London

3. Gambirasio R, Riand N, Stern R, Hoffmeyer P (2001) Total elbow replacement for complex fractures of the distal humerus. An option for the elderly patient. J Bone Joint Surg Br 83(7):974-978

4. Helfet DL, Hotchkiss RN (1990) Internal fixation of the distal humerus: a biomechanical comparison of methods. J Orthop Trauma 4(3):260-264

5. Helfet DL, Schmeling GJ (1993) Bicondylar intraarticular fractures of the distal humerus in adults. Clin Orthop 292:26-36

6. Huang TL, Chiu FY, Chuang TY, Chen TH (2004) Surgical treatment of acute displaced fractures of adult distal humerus with reconstruction plate. Injury 35(11):1143-1148

7. Jupiter JB (1995) Complex fractures of the distal part of the humerus and associated complications. Instr Course Lect 44:187-198

8. Kundel K, Braun W, Wieberneit J, Ruter A (1996) Intraarticular distal humerus fractures. Factors affecting functional outcome. Clin Orthop 332:200-208

9. Moneim MS, Garst JR (1995) Vascular injuries associated with elbow fractures and dislocations. Int Angiol 14(3):307-312

10. Regel G, Seekamp A, Blauth M et al (1996) Complex injury of the elbow joint. Unfallchirurg 99(2):92-99

11. Ring D, Jupiter JB (2000) Fractures of the distal humerus. Orthop Clin North Am 31(1):103-113

12. Robinson CM, Hill RM, Jacobs N et al (2003) Adult distal humeral metaphyseal fractures: epidemiology and results of treatment. J Orthop Trauma 17(1):3847

13. Weber O, Burger C, Kabir K et al (2009) Primary endoprosthetic replacement of fractured elbow in elderly patients. Unfallchirurg 112(9):778-784

14. Wenzl ME, Raimund F, Fuchs S et al (2007) Distale Humerusfrakturen. Trauma Berufskrankh [Suppl 2] 9:183-191 